\title{
Hypertension and renal disease
}

\section{The histology of eclamptic lesions}

\author{
A. D. T. GOVAN
}

From the Research Department, Royal Maternity Hospital, Glasgow

The following is a brief account of the main lesions found in fatal eclampsia with a discussion of their histogenesis and relationship to preeclampsia. It is based on a survey of 50 cases.

In general it may be said that eclampsia is characterized by haemorrhagic lesions, usually small, in the main parenchymatous organs. The haemorrhages may on occasion be large. This is common in the brain, infrequent in the adrenals, and, only rarely, a large subcapsular haemorrhage may be found on the upper surface of the right lobe of the liver. It is interesting to note that although small haemorrhages are commonly found in the liver, they do not occur with any regularity in other organs whose blood supply is part of the portal system. Similarly, although haemorrhages may be found in the adrenals they are almost never found in the kidneys.

\section{Liver}

The hepatic lesions are said to be pathognomonic of eclampsia. In approximately one-third of cases, however, they are absent. The liver is normal to the naked eye and histologically the structure is maintained. Closer examination generally reveals that the cell columns are loosened. The hepatic arteriole is of normal calibre and there may be a hyaline subendothelial deposit in the wall.

Other cases show distinctive lesions which vary in type and severity. The variations may all be exhibited in one liver but most commonly the changes are remarkably uniform in individual cases. Anoxic lesions form one group of these lesions. The minimum change consists of small areas of necrobiosis in the midzones of the lobules. More severe lesions, causing necrosis of most of a liver lobule, leaving only a narrow viable zone, some five or six cells deep around the portal tract, are occasionally seen. In exceptional circumstances the necrosis may become confluent involving several lobules. Small thrombi may be found in the hepatic arterioles but most commonly these vessels are dilated. Sometimes there is a loss of structure in the walls of these vessels. These extensive necrotic changes rarely occur in uncomplicated eclampsia. Most often there has been an episode of severe clinical haemorrhage at or near the eclamptic phase.

Occasionally centrolobular necrosis may be found in association with other lesions considered to be more typical of eclampsia - circumscribed periportal lesions (fig 1). In these cases it is difficult to determine whether the centrolobular necrosis is the result of the periportal lesions, but again in many such cases eclampsia has been complicated by the occurrence of a haemorrhagic episode such as abruptio.

Periportal lesions are basically of two types. In the first there are small lake haemorrhages formed among the liver cells of several columns immediately

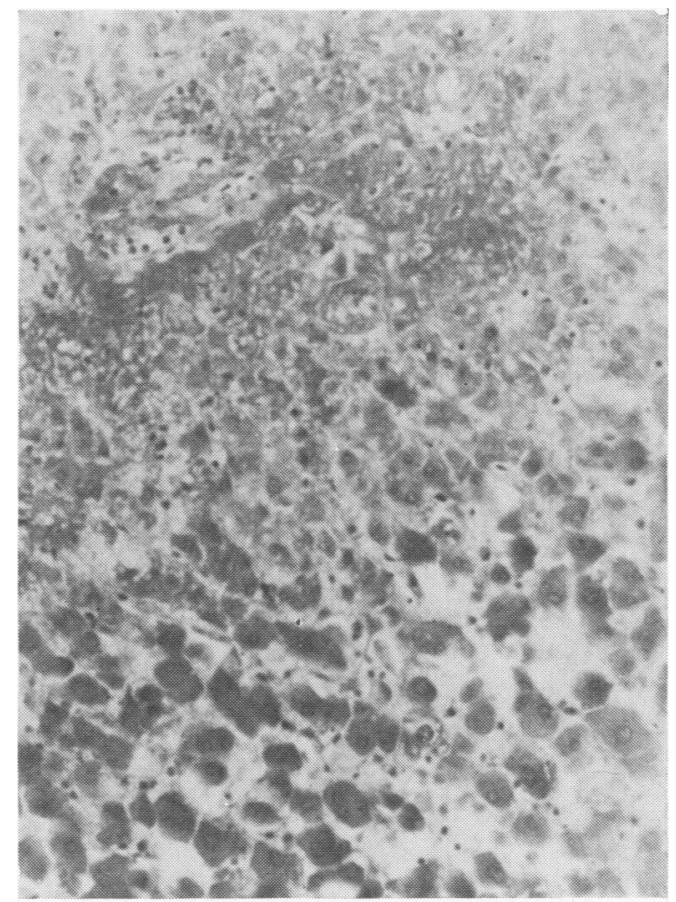

Fig 1 Liver showing periportal lesion plus centrolobular necrosis. $H \& E \times 200$. 
adjacent to the portal tract. These compress the liver cells causing necrosis. Sometimes haemorrhage is present within the portal tract itself (fig 2). The hepatic arteriole is commonly dilated and there is a complete loss of structure in its wall suggesting possible necrosis.

The second type of periportal lesion is fibrinous, affecting an area similar in size and situation to the haemorrhagic lesion; skeins of fibrin entangle the liver cells and fill the sinusoids. The hepatic arterioles are frequently thrombosed and necrotic (fig 3). According to some these two types of periportal lesion are merely two stages in the one process.

\section{Brain}

Lesions may be found in the grey matter of the cortex, the subcortical white matter, deeper white mat- ter of the hemispheres, basal ganglia, midbrain and pons. The lesions are essentially of two types. One is haemorrhagic and can frequently be seen with the naked eye. They vary in size. In the cortical grey matter they are petechial in type. Similar lesions may be seen in the other areas mentioned but very much larger haemorrhages occur in the cortical white matter, basal ganglia and pons.

The second type of lesion is most commonly of microscopical size and is in the nature of an infarct. These are usually associated closely with the smaller haemorrhagic lesions.

Small haemorrhages may be confined to exudation of blood into the Virchow-Robin space resulting in cuffing of the small vessels. Others are larger and extend into the surrounding brain matter (fig 4). Nearby small vessels are frequently thrombosed. In most cases small softenings are found near these

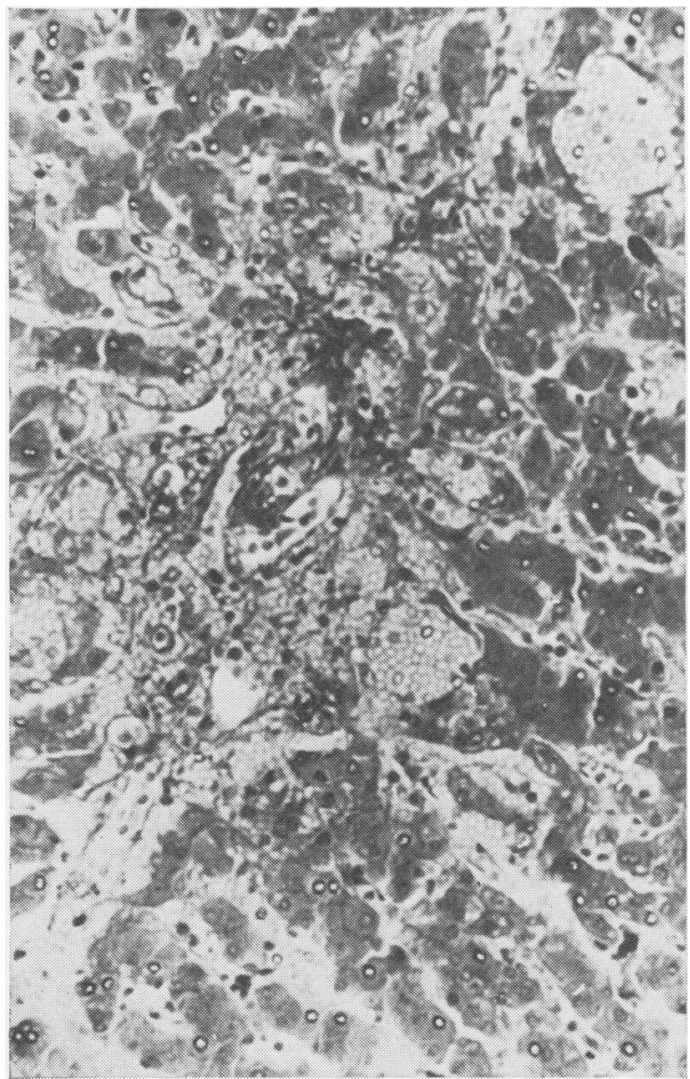

Fig 2 Haemorrhagic type of periportal lesion. Blood lakes are formed and the liver cells are compressed. $H \& E \times 200$.

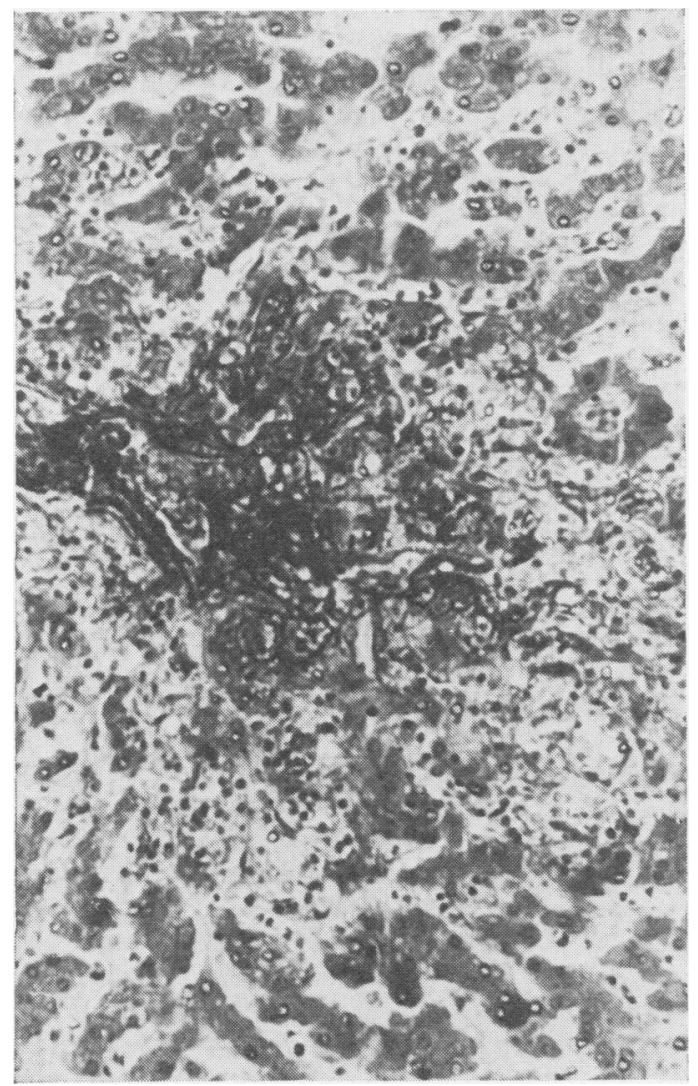

Fig 3 Fibrinous periportal lesion. A tangle of fibrin involves the liver cells around the portal tract. $H \& E$ $\times 200$. 


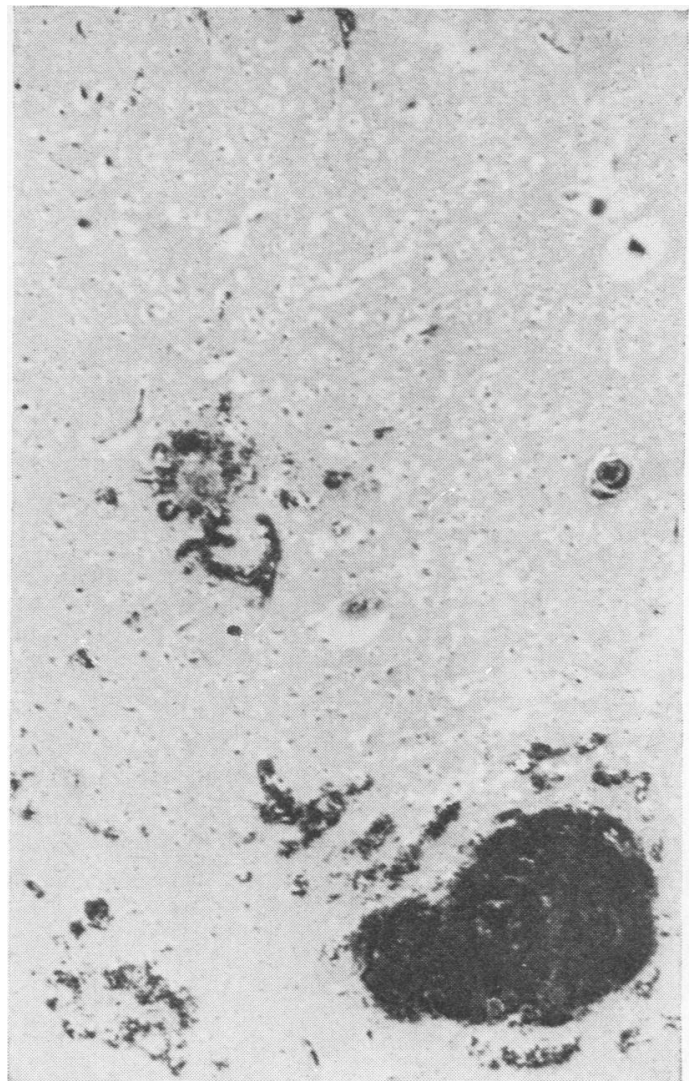

Fig 4 Perivascular haemorrhages in the brain. One is larger and has spread into the surrounding brain substance. $H \& E \times 100$.

small haemorrhages. The small vessels related to the softenings are also thrombosed and more often than not the vessel walls are destroyed (fig 5).

Large haemorrhages are always in the deeper parts of the brain. Thrombosed vessels are found at the periphery of these haemorrhages, but it is impossible to determine whether the lesion is of progressive nature initiated by a thrombotic process with subsequent haemorrhage into a softened area, or primarily haemorrhagic with secondary thrombosis.

\section{Kidney}

Unlike the brain, liver and adrenals, visible haemorrhages are not seen in the kidneys which at necropsy are moderately swollen and pale. This is reflected in the bloodless appearance of the cortex under the microscope. The glomeruli are prominent because of their enlargement, often filling the capsular spaces and projecting into the neck of the first convoluted tubule. They have a rounded shape with blunt lobulations and appear to be more solid than usual (fig 6). This apparent solidity is due to prominence of the interstitial ground substance or mesangium, and in most instances a reduction in the lumina of the capillaries. In many instances the capillaries are reduced to mere slits, due to swelling of the endothelial cells. There is no evidence of any change in the basement membrane of these capillaries (fig 7). The epithelial cells covering the lobules are also swollen. Hyaline droplets, staining strongly with fuchsin, are found in the mesangium, in epithelial cells covering the glomerulus and in the epithelium of the proximal convoluted tubule. Flecks of fat are found in the same situations.

Several other changes may be found but are less constant. Sometimes a glomerulus shows excessive dilatation and congestion of the capillary loops. Hyaline thrombus substance may be found in

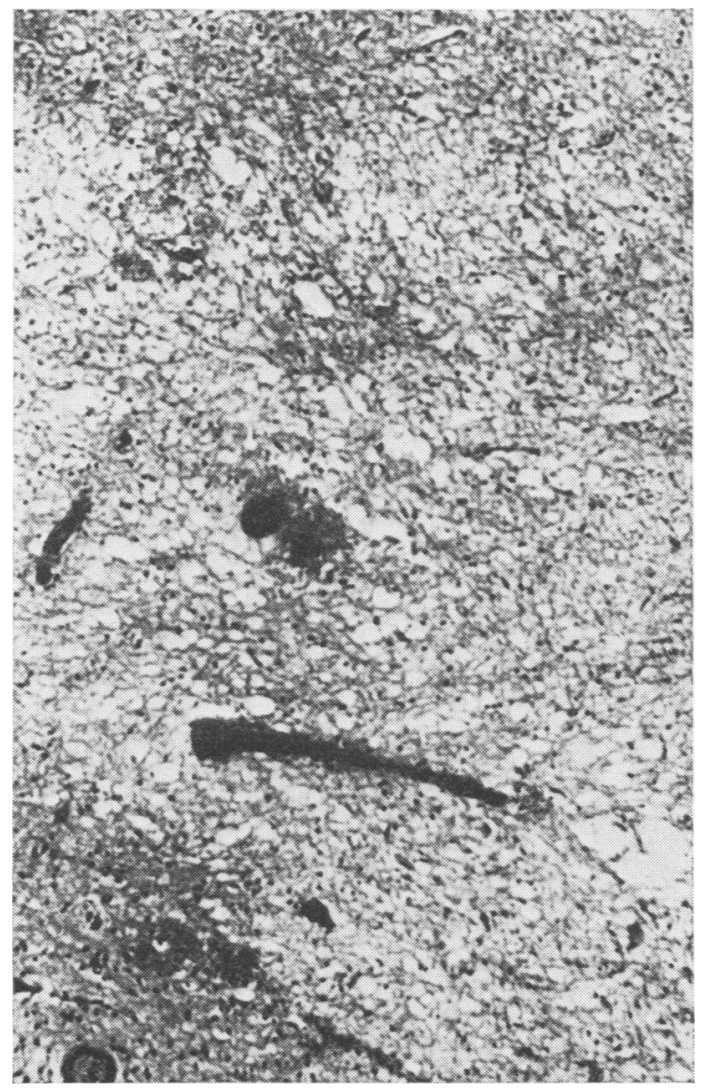

Fig 5 Area of brain softening with thrombosis of the associated vessels. $H \& E \times 100$. 


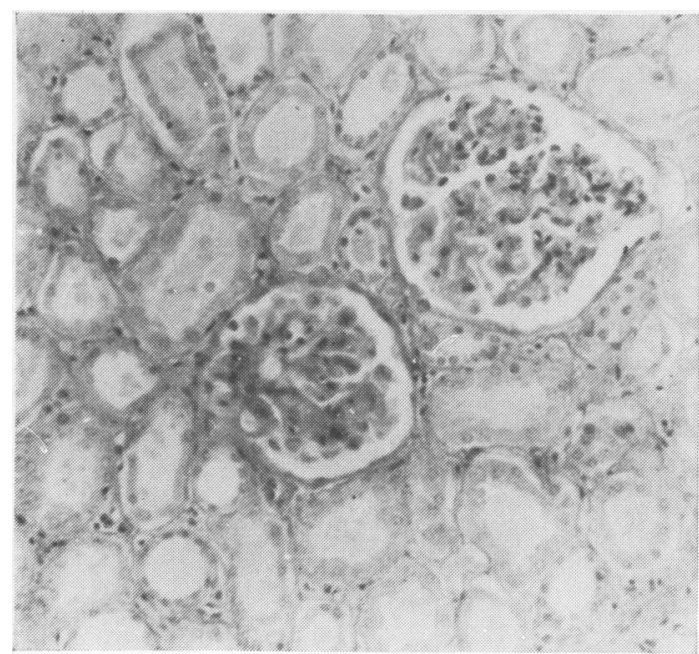

Fig 6 Kidney showing enlarged bloodless glomeruli with blunt lobulations. $H \& E \times 200$.

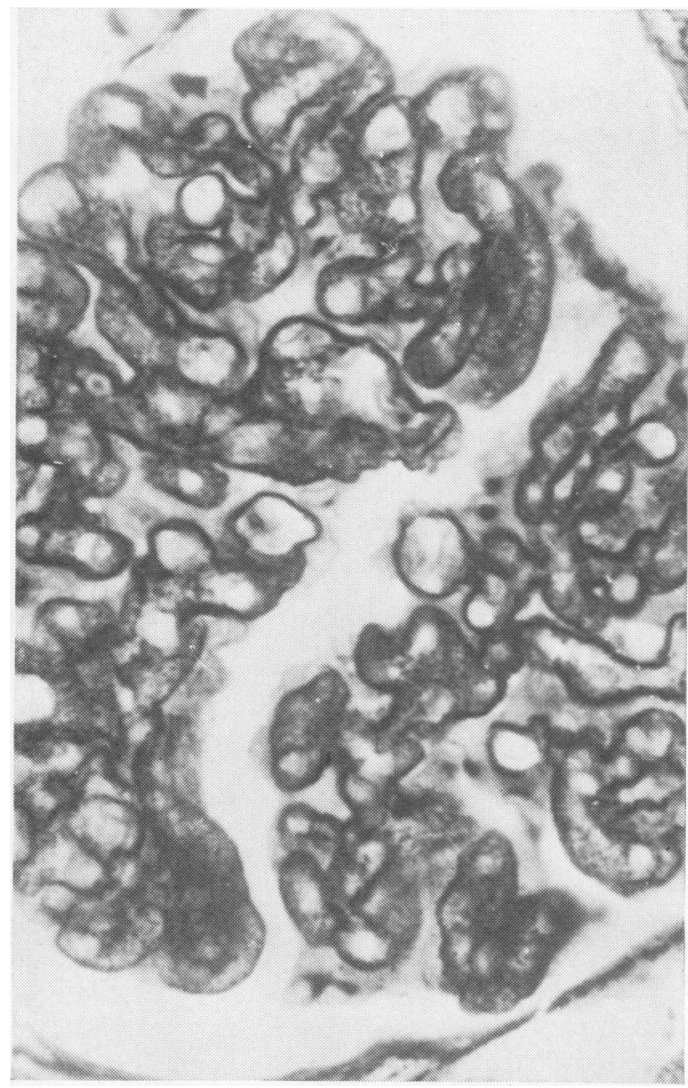

Fig 7 High-power view of glomerulus stained to show basement membranes. These are normal. Granular deposits can be seen in the cytoplasm of cells. PASmethenamine $\times 650$. glomerular capillary loops (fig 8) and in an odd glomerulus thrombus material may extend from the afferent arteriole into the capillaries. Fuchsinophile deposits are quite commonly found beneath the endothelium of afferent arterioles and arcuate arteries. In some of the glomeruli where thrombotic material has occluded the capillaries of a lobule, the whole lobule has a club shape. The nuceli, endothelial and epithelial, are crowded together giving an appearance of increased cellularity.

\section{Heart}

When the patient dies in the eclamptic phase or shortly after, the only changes to be seen in the heart consist of subendocardial haemorrhages in the wall of the left ventricle. These extend into the adjacent muscle fibres. In patients dying some days later the adjacent myocardial fibres can be seen to have undergone degeneration with loss of striations and hyalinization. Other and more important lesions are to be found in the inner half of the myocardium. These are only found on microscopical examination. Fuchsin stains are useful in picking up these changes, which progress from focal loss of staining in the fibres (fig 9) in the early stages of disappearance of striations and conversion of the fibres into amorphous material. At a later stage still attempts at healing take place with proliferation of nuclei.

\section{Lungs}

Frequently small haemorrhages may be found in the lung substance, most commonly towards the periphery. Groups of alveoli are filled with red cells. No constant change can be found in the blood vessels. Occasionalsmall fibrinous thrombi may be found in the pulmonary vessels but they are inconstant and not always associated with the haemorrhagic lesions.

\section{Spleen}

Haemorrhages are common in the splenic pulp. In addition the lymphoid tissue around branches of the arterial vessels is often necrotic and infiltrated with fibrin.

\section{Adrenals}

Streak haemorrhages are common in the adrenal cortex. These are of capillary type. Very occasionally larger haemorrhages, causing disruption of parts of the cortex, may be found. Thrombosis may also be found in adjacent vessels but this may be secondary to the haemorrhage. 


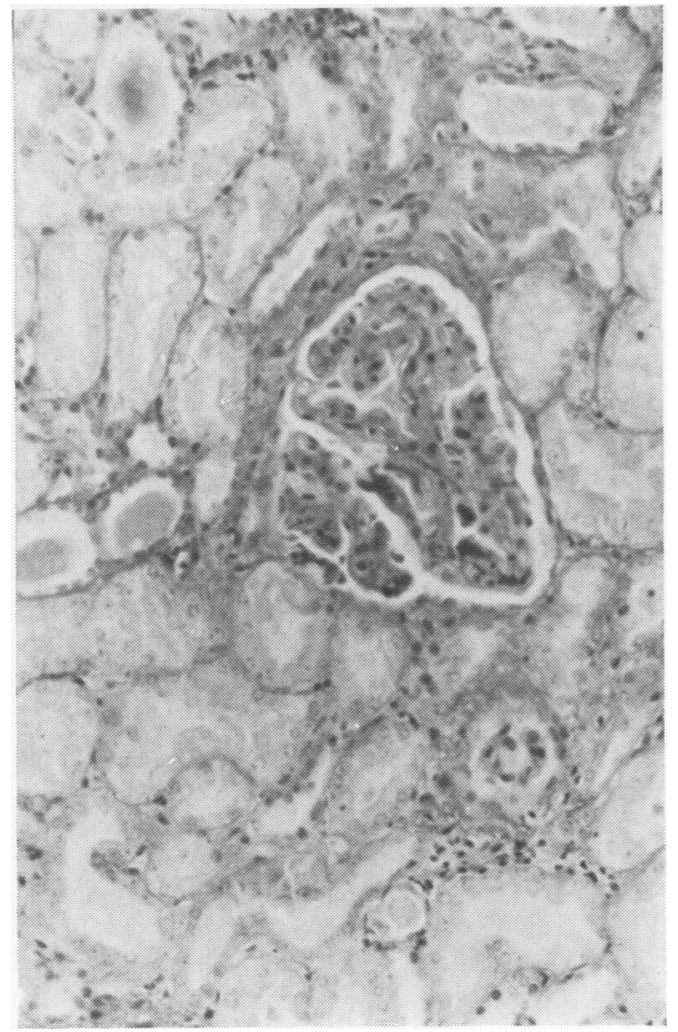

Fig 8 Glomerulus showing small hyaline thrombi in capillary loops. Picro Mallory $\times 200$.

\section{The Anatomy of Eclamptic Lesions}

In most of the organs the changes consist of haemorrhage, thrombosis and cell necrosis. The distribution of these changes is difficult to explain in many instances. Hypoxia is the obvious cause of centrolobular necrosis in the liver. As has been noted, this change, when extensive, is usually associated with eclampsia complicated by an intercurrent clinical haemorrhage. The periportal fibrinous and haemorrhagic lesions are essentially focal and suggest an entirely locally acting agent. Similarly in the brain the site of the lesions suggests a relationship to vascular supply. Some of them in the cortex occur in the so-called 'watershed' areas where the anastomotic blood supply is of capillary type and these areas suffer when there is a general reduction in blood flow such as occurs with clinical haemorrhage. The other lesions, especially those occurring in the white matter are again the result of a local vascular incident either occlusive or haemorrhagic; which of these alternatives is not quite clear.

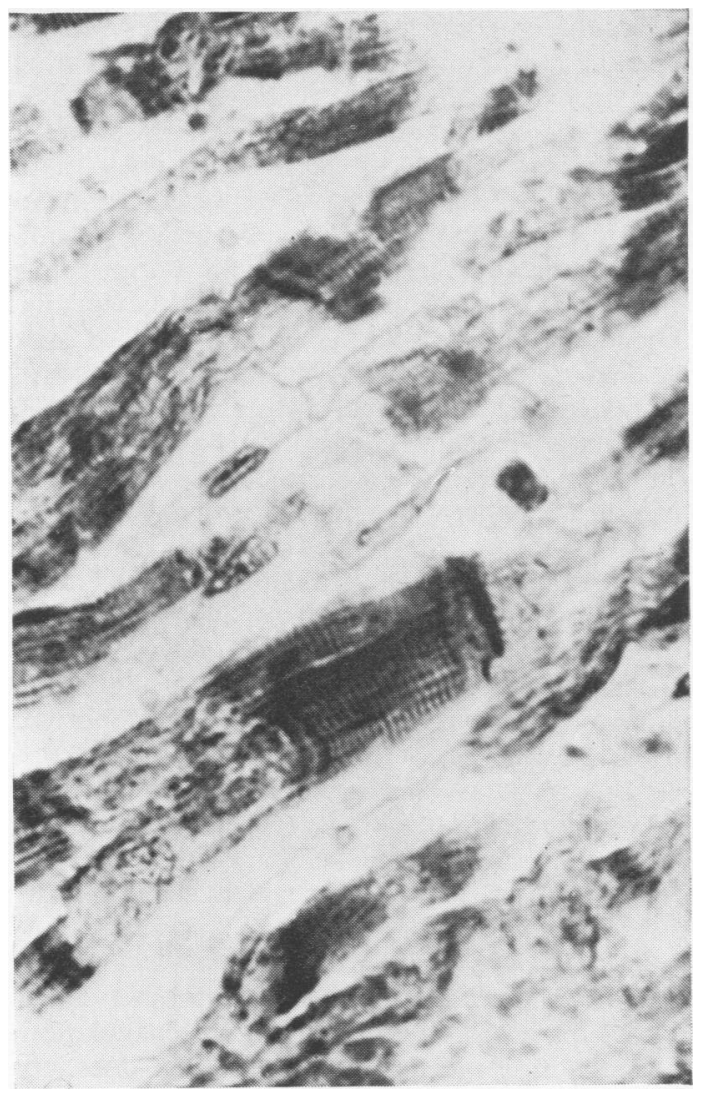

Fig 9 Section of myocardium from patient who died 48 hours after eclamptic fits. The fibres show loss of staining and conversion of their substance into $a$ granular amorphous substance. $H \& E \times 650$.

Although marked changes are found in certain organs, the lack of lesions in others is puzzling. The liver and the intestines have been cited as an example of this. The cause might be thought to lie in the possession of a portal blood supply in the case of the liver, but a portal system also supplies the pituitary gland and it is almost never affected in eclampsia.

The fact that the kidney does not show the same haemorrhagic lesions as are found in the other organs must be related to the quite separate method of control of renal blood flow in relation to blood pressure.

\section{Discussion}

The above is a summary of the lesions found in eclampsia. For more detailed pathological descriptions the reader is referred to Govan $(1954,1961)$ and Sheehan and Lynch (1973). 
It is apparent that many of the changes seen in these fatal cases are acute and therefore peculiar to the eclamptic phase. They are both haemorrhagic and thrombotic in nature and the main question is whether either of them is in any way relevant to the preeclampsia phase. At the present moment attention is focused upon the importance of thrombotic phenomena. It has been known for a long time that eclampsia is accompanied by thrombocytopenia and depletion of coagulation factors (Pritchard et al, 1954) and that the level of fibrin degradation products reaches a high level (Morris et al, 1964). Subsequently Henderson et al (1970) have found that fibrin degradation products are increased in preeclampsia, and this was confirmed by Bonnar et al (1971). Howie et al (1971) also demonstrated raised fibrin degradation products in preeclampsia. These findings have been interpreted as evidence of disseminated intravascular coagulation, in eclampsia at least. Unfortunately it is difficult to demonstrate thrombi in any considerable numbers in post-mortem material. McKay et al (1953) did report widespread fibrin thrombi in fatal eclampsia but this has not been the experience of other observers. It has been suggested that the fibrin is attacked by lytic enzymes and therefore the thrombi are not evident histologically but Bonnar et al (1971) have reported a depression of fibrinolytic activity in these cases. This requires confirmation and there is also a need for a stricter definition of disseminated intravascular coagulation. Increase in fibrin degradation products does not always indicate that the thrombotic process is disseminated in the sense which the phrase usually implies.

Histological study of the organs in the preeclamptic phase has been made in only a few instances. Sheehan and Lynch (1973) have made a detailed and extensive study of post-mortem material, but only a few attempts have been made to obtain biopsy material from patients. The descriptions given of changes in the liver are unsatisfactory. Maqueo et al (1964) record fatty change and focal necrosis but there is no mention of thrombotic changes. Renal biopsies have been studied in a larger number of cases (Pollak and Nettles, 1960; Pirani et al, 1963; Thaler, 1970). These studies confirm the narrowing of the glomerular capillaries, absence of change in the basement membranes and also indicate that there is no significant change in the epithelial foot processes. No mention is made of haemorrhages or of any great frequency of thrombosis. There is, however, a fairly constant finding of fibrinous material and lipid beneath the endothelium of the glomerular capillaries.

The absence of haemorrhages and the infrequent finding of thrombi in the renal vessels seems to me to be significant. The kidney is protected to a great extent from the physical effects of hypertension by its local regulation of its circulation. Nevertheless there is evidence of protein material, partly fibrinogen or fibrin, passing through the glomerular capillaries. A possible part interpretation of the lesions described may be that there is not only an increased coagulability of the blood but also an increased permeability of blood vessels which would allow blood protein constituents to filter through vessel walls. This would account for the deposition of protein in the glomerular apparatus. The thrombotic and haemorrhagic lesions in other organs might be explained on the basis of hypertension and increased coagulability of the blood, the damage produced by the hypertension determining the site of thrombus deposition.

\section{Summary}

A brief description is given of the main lesions found in eclampsia. Emphasis is placed on the marked difference between the type of lesion found in the kidney and those found in other organs. It is suggested that they may be explained on the basis of increased coagulability of the blood, hypertension and possibly increased permeability of blood vessels. Hypertension may damage vessels and determine the sites of thrombus deposition. Increased permeability of blood vessels in the kidney probably accounts for the appearance of protein deposits in the mesangium of the glomerulus.

\section{References}

Bonnar, J., McNicol, G. P., and Douglas, A. S. (1971). Coagulation and fibrinolytic systems in pre-eclampsia and eclampsia. Brit. med. J., 2, 12-16.

Govan, A. D. T. (1954). Renal changes in eclampsia. J. Path. Bact., 67, 311-322.

Govan, A. D. T. (1961). The pathogenesis of eclamptic lesions. Path. et Microbiol. (Basel), 24, 561-575.

Henderson, A. H., Pugsley, D. J., and Thomas, D. P. (1970). Fibrin degradation products in pre-eclamptic toxaemia and eclampsia. Brit. med.J., 3, 545-547.

Howie, P. W., Prentice, C. R. M., and McNicol, G. P. (1971). Coagulation, fibrinolysis and platelet functions in preeclampsia, essential hypertension and placental insuffciency. J. Obstet. Gynaec. Brit. Cwlth, 78, 992-1003.

McKay, D. G., Merrill, S. J., Weiner, A. E., Hertig, A. T., and Reid, D. E. (1953). The pathologic anatomy of eclampsia, bilateral renal cortical necrosis, pituitary necrosis, and other actue fatal complications of pregnancy, and its possible relationship to the generalized Shwartzman phenomenon. Amer. J. Obstet. Gynec., 66, 507-539.

Maqueo, M., Azuela, J. C., and Dosa de la Vega, M. (1964). Placental pathology in eclampsia and pre-eclampsia. Obstet. and Gynec., 24, 350-356.

Morris, R. H., Vassali, P., Beller, F. K., and McCluskey, R. T. (1964). Immunofluorescent studies of renal biopsies in the diagnosis of toxemia of pregnancy. Obstet. and Gynec., 24, 32-46 
Pirani, C. L., Pollak, V. E., Lannigan, R., and Folli, G. (1963). The renal glomerular lesions of pre-eclampsia: electron microscopic studies. Amer. J. Obstet. Gynec., 87, 1047-1070.

Pollak, V. E., and Nettles, J. B. (1960). The kidney in toxemia of pregnancy: a clinical and pathologic study based on renal biopsies. Medicine (Baltimore), 39, 469-526.

Pritchard, J. A., Weisman, R., Jr., Ratnoff, O. D., and Vosburgh, G. J. (1954). Intravascular hemolysis, throm- bocytopenia and other hematologic abnormalities associated with severe toxemia of pregnancy. New Engl.J. Med., 250, 89-98.

Sheehan, H. L., and Lynch, J. B. (1973). Pathology of Toxaemia of Pregnancy. Churchill-Livingstone, Edinburgh and London.

Thaler, H. (1970). Nierenveränderungen bei Schwangerschaft. Wien. klin. Wschr., 82, 421-425. 